ISSN 1991-8631

Original Paper

http://indexmedicus.afro.who.int

\title{
Evaluation de la connaissance et utilisation des variétés de piment (Capsicum) cultivées en Côte d'Ivoire
}

\author{
Clément KOUASSI KOUASSI ${ }^{1,2}$ et Rose KOFFI-NEVRY ${ }^{1 *}$ \\ ${ }^{1}$ Laboratoire de Biotechnologie et de Microbiologie Alimentaire de l'UFR des Sciences et Technologies des \\ Aliments de l'Université d'Abobo-Adjamé, 02 BP 801 Abidjan 02, Côte d'Ivoire. \\ ${ }^{2}$ Laboratoire National de la Santé Publique, 18 BP 2403 Abidjan 18, Côte d'Ivoire. \\ *Auteur correspondant, E-mail : rosenevry2002@yahoo.fr; Phone: + 22507688334.
}

\section{RESUME}

Cette étude a été entreprise pour répertorier et décrire les différentes variétés de piment consommées en Côte d'Ivoire en vue de leur valorisation. Un questionnaire a permis de recueillir des renseignements sur la connaissance et les différentes formes d'usages du piment. Les variétés de Capsicum étudiées sont connues à 98\% aussi bien par les femmes, les hommes que par les différents groupes ethniques. Les 2 espèces cultivées et retrouvées sur les marchés d'Abidjan sont Capsicum annuum et Capsicum frutescens, réparties en 6 variétés: Capsicum annuum jaune, Capsicum annuum antillais, Capsicum frutescens soudanais, Capsicum frutescens attié, Capsicum frutescens doux et Capsicum frutescens oiseau. Le piment est consommé quotidiennement à $97,16 \%$ chez les femmes contre $87 \%$ chez les hommes pour ses propriétés nutritionnelle $(18 \%)$ et sensorielle $(15 \%)$. Il est consommé et apprécié pour son goût piquant $(23 \%)$ moyennement fort (32\%), suivi de l'arôme (10\%). Les sauces sont les modes de consommation les plus fréquentes $(30,24 \%)$ suivies par les grillades $(8,89 \%)$. Le piment est aussi connu par les populations pour ses propriétés médicinales. La constipation serait à $23 \%$, l'affection la plus traitée par les variétés de Capsicum, suivie des gastro-entérites (14\%). Ces informations recueillies sur le piment font de ce fruit-légume, une ressource agricole à prendre en compte dans tout programme de valorisation des richesses floristiques dont disposent la Côte d'Ivoire.

(C) 2012 International Formulae Group. All rights reserved.

Mots clés : Capsicum, piment, épice, propriété sensorielle, propriété médicinale.

\section{INTRODUCTION}

L'intérêt des plantes légumières pour l'alimentation des populations est très largement reconnu dans le monde (Ludy \& Mattes, 2011; Zimmer et al., 2012). Ce sont toutes les plantes telles le piment dont les feuilles, les fruits et les racines sont utilisés dans la préparation des sauces (FAO, 2006). Le piment appartient au genre Capsicum, de la famille Solanaceae, laquelle inclut d'autres légumes ayant une valeur économique importante. C'est un fruit tropical originaire de l'Amérique (Sud et Centrale), puis disséminé en Europe, en Afrique et en Asie (Menichini et al., 2009 ; Zimmer et al., 2012). Les espèces les mieux connues et utilisées comme épices dans les régions tropicales et subtropicales sont Capsicum аппиит et 
Capsicum frutescens (Tano et al., 2008 ; Menichini et al., 2009). Capsicum annuum présente une grande variété de formes cultivées et renferme des variétés piquantes et non piquantes telles le poivron. Capsicum frutescens a les variétés dont les formes sont plus petites et généralement piquantes (Jouzier, 2005).

Dans le monde entier, la consommation des fruits de Capsicum probablement classés parmi les premiers épices ou additifs alimentaires, ne cesse d'augmenter. La Chine est le plus grand producteur de piment avec plus de 125000000 tonnes en 2005. Ainsi, en 2005, l'Asie a produit $65,6 \%$ de piment, suivie de l'Amérique (13,9\%), et de l'Afrique $(8,8 \%)$. En Afrique, le Nigeria, l'Egypte et le Ghana ont une production régulière qui se retrouve sur le marché international (FAO, 2006). Le piment est un produit de base de cuisines ethniques comme la cuisine thaïlandaise, coréenne, indienne, hongroise, africaine, mexicaine etc. (Kollmannsberger et al., 2011). Les fruits de Capsicum sont très appréciés un peu partout dans le monde. Ils sont essentiels dans les mets africains, en particulier dans les mets ivoiriens tels le kédjénou et le biokesseu qui sont des soupes très épicées (Kouassi, 2012). Le piment peut être consommé à l'état frais de manière directe, frit, en sauce ou en conserve. Il est souvent associé en mélange avec divers autres légumes. A l'état déshydraté, le piment est utilisé sous forme de poudre et sert dans les assaisonnements des plats, des viandes braisées etc. (Tano et al., 2008). Les oléorésines qui sont des extraits de fruits secs tels le piment (paprika, chili...) sont utiles dans la production de certains aliments et comme colorant de divers produits (Meghvansi et al., 2010).

De nombreuses populations connaissent le grand potentiel et l'action stimulante des piments qui rehaussent la saveur des aliments, généralement les viandes, les céréales et les sauces fades, en augmentant la salivation et la sensation de chaleur dans la bouche (Kouassi, 2012). Le piment peut être même un confort post-digestion. En revanche, beaucoup de personnes ignorent sa composition, le complément appréciable de calorie, de vitamines, de fibres, de sels minéraux et de protéines que le piment peut apporter dans l'alimentation. Connus aussi comme plante médicinale, les fruits de Capsicum sont employés en médecine traditionnelle pour leurs propriétés antimicrobiennes dues aux métabolites secondaires qu'ils contiennent (HervertHernandez et al., 2010; Kouassi et al., 2010).

En Côte d'Ivoire, la vulgarisation et la sauvegarde des ressources phytogénétiques locales nécessitent l'existence d'une base de données actualisée sur leur connaissance, leur diversité, leur distribution, leur mode d'usage et leurs considérations socioculturelles. Le recours des populations ivoiriennes aux différentes variétés de Capsicum dans leur alimentation et leurs usages dans la médecine traditionnelle nécessite d'entreprendre des études en vue de l'établissement d'une base de données sur les variétés de piment utilisées en Côte d'Ivoire. L'objectif de cette étude vise à répertorier et à décrire les différentes variétés de piment cultivées et consommées, à travers une collecte de renseignements auprès des consommateurs.

\section{MATERIEL ET METHODES}

Enquête sur la connaissance et la consommation du piment en Côte d'Ivoire

Une enquête a été réalisée dans les différentes communes de la ville d'Abidjan (Côte d'Ivoire) afin de recueillir des renseignements sur la connaissance, les différentes formes d'usages, les raisons de la consommation du piment etc., et de cerner son importance dans l'alimentation de la population ivoirienne. A cet effet, un questionnaire a été élaboré en tenant compte 
des différents groupes ethniques de la Côte d'Ivoire à savoir, les Akan (144), les Krou (93), les Mandé (82), les voltaïques (100), et les immigrants des pays du nord de la Côte d'Ivoire (Burkina Fasso, Mali, et Niger) (47) et ceux des autres pays africains.

L'enquête qui a duré 12 mois a concerné des vendeuses de piment dans les grands marchés, des grossistes, des consommateurs, des restauratrices et des vendeurs de médicaments traditionnels. Elle s'est déroulée dans les dix communes de la ville d'Abidjan: Plateau, Treichville, Marcory, Koumassi, Port-Bouet, Cocody, Abobo, Adjamé, Attécoubé et Yopougon. Les répondants sont des deux sexes, de tous les niveaux d'étude et de toutes les couches sociales. Le seul critère d'exclusion était l'âge minimum de 15 ans. Au total, l'enquête a porté sur 506 personnes. Les différentes variétés de piments ont été ensuite identifiées au Centre National de Floristique de l'Université de Cocody, Abidjan. Les renseignements recueillis des répondants ont été exprimés en pourcentages.

\section{Analyse des données de l'enquête}

Les différentes données obtenues ont été traitées avec des logiciels statistiques SPSS 11.05. Des recodages ont été effectués pour leur exploitation. Ces données ont été transmises sur Excel et les résultats présentés sous forme de graphique.

\section{RESULTATS}

Le Tableau 1 présente les caractéristiques et usages des variétés de piment retrouvées dans les marchés d'Abidjan. Deux espèces à savoir, Capsicum annuum et Capsicum frutescens sont réparties en 6 variétés: Capsicum a. jaune, Capsicum a. antillais, Capsicum fr. soudanais, Capsicum fr. attié, Capsicum fr. doux et Capsicum fr. oiseau. Sur les 6 variétés répertoriées, seule
Capsicum fr. oiseau n'est pas une espèce cultivée. Capsicum a. jaune et antillais sont utilisées principalement dans les sauces ou émincées pour accompagner les grillades. Capsicum fr. attié, doux et oiseau sont utilisées dans la médecine traditionnelle ou sous forme de poudre pour assaisonner les plats. Les variétés de $C$. annuum sont généralement destinées à la consommation alors que celles de $C$. frutescens sont utilisées principalement dans les médicaments traditionnels (Tableau 1).

Les résultats de la Figure 1 indiquent que $99,23 \%$ des femmes connaissent le piment avec une consommation quotidienne de $97 \%$, alors que tous les hommes (100\%) connaissent le piment mais le consomme à seulement $87 \%$. En dehors des Akan (97,22\%) et des Mandés (98,78\%), les autres groupes ethniques connaissent le piment à $100 \%$. (Figure 2). La Figure 3 montre que le piment est préférentiellement consommé pour son « goût piquant ou brûlant» $(23 \%)$, suivi de la couleur (10\%) et enfin de l'arôme (7\%). Lorsque ces propriétés sont associées, le piment est essentiellement consommé à $21 \%$ pour ces trois propriétés. La Figure 4 illustre les propriétés qui soutiennent la consommation de ce fruit-légume. Elle indique que le piment est d'abord consommé pour ses propriétés nutritionnelles (18\%), suivi des propriétés sensorielles (15\%), vitaminiques $(9 \%)$ et enfin des propriétés antiseptiques (7\%). Les sauces sont les modes de consommation les plus fréquentes $(30,24 \%)$ suivies par les grillades $(8,89 \%)$ (Figure 5).

La Figure 6 montre que la constipation (23\%), des gastro-entérites (14\%), des infections (7\%), des angines (5\%) et le rhumatisme $(4 \%)$ sont des affections traitées par le piment. 
Tableau 1 : Caractéristiques et usages des variétés de piments retrouvées dans les marchés d'Abidjan.

\begin{tabular}{|c|c|c|c|}
\hline Nom scientifique & $\begin{array}{l}\text { Variété et nom } \\
\text { vernaculaire }\end{array}$ & $\begin{array}{l}\text { Caractéristiques et } \\
\text { description à } \\
\text { maturité }\end{array}$ & $\begin{array}{l}\text { Usages et } \\
\text { consommation }\end{array}$ \\
\hline *Capsicum annuum & jaune Burkina & Carré charnu jaune & $\begin{array}{l}\text { Sauces, pâtes de } \\
\text { légumes. }\end{array}$ \\
\hline Capsicum annuum & jaune Burkina & Carré (petit) jaune & $\begin{array}{l}\text { Sauces, pâtes de } \\
\text { légumes. }\end{array}$ \\
\hline Capsicum annuum & jaune Burkina & $\begin{array}{l}\text { Long (charnus) } \\
\text { jaune }\end{array}$ & $\begin{array}{l}\text { Sauces, pâtes de } \\
\text { légumes. }\end{array}$ \\
\hline Capsicum annuum & jaune Burkina & Long (petit) jaune & $\begin{array}{l}\text { Sauces, pâtes de } \\
\text { légumes. }\end{array}$ \\
\hline *Capsicum annuum & antillais & $\begin{array}{l}\text { Carré (charnus) } \\
\text { rouge }\end{array}$ & $\begin{array}{l}\text { Sauces, pâtes ou frais } \\
\text { en accompagnement } \\
\text { d'autres plats. }\end{array}$ \\
\hline Capsicum annuum & antillais & Carré (petit) rouge & $\begin{array}{l}\text { Sauces, pâtes ou frais } \\
\text { en accompagnement } \\
\text { d'autres plats. }\end{array}$ \\
\hline Capsicum annuum & antillais & $\begin{array}{l}\text { Long (charnus) } \\
\text { rouge }\end{array}$ & $\begin{array}{l}\text { Sauces, pâtes de } \\
\text { légumes. }\end{array}$ \\
\hline *Capsicum frutescens & soudanais & $\begin{array}{l}\text { Court conique } \\
\text { rouge }\end{array}$ & $\begin{array}{l}\text { Seul ou associé comme } \\
\text { médicament } \\
\text { traditionnel. Sous } \\
\text { forme de poudre pour } \\
\text { assaisonnement. }\end{array}$ \\
\hline *Capsicum frutescens & attié & Effilé rouge & $\begin{array}{l}\text { Seul ou associé comme } \\
\text { médicament } \\
\text { traditionnel. Sous } \\
\text { forme de poudre pour } \\
\text { assaisonnement. }\end{array}$ \\
\hline Capsicum frutescens & attié & Large rouge & $\begin{array}{l}\text { Seul ou associé comme } \\
\text { médicament } \\
\text { traditionnel, sous forme } \\
\text { de poudre pour } \\
\text { assaisonnement. }\end{array}$ \\
\hline *Capsicum frutescens & doux & Effilé rouge & Préparation des sauces \\
\hline Capsicum frutescens & $\begin{array}{l}\text { Oiseau (non } \\
\text { cultivé) }\end{array}$ & Effilé (très petit) & $\begin{array}{l}\text { Seul ou associé comme } \\
\text { médicament } \\
\text { traditionnel. }\end{array}$ \\
\hline
\end{tabular}

(*) Les variétés de Capsicum les plus utilisées 


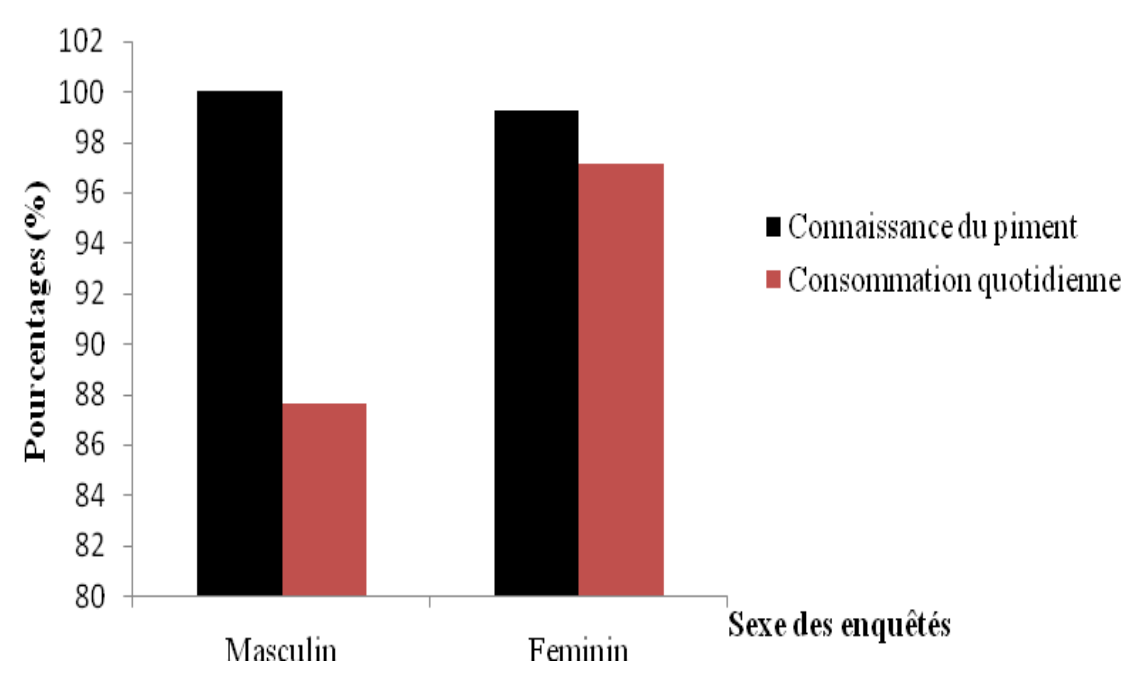

Figure 1 : Répartition de la connaissance et de la consommation du piment selon le sexe.

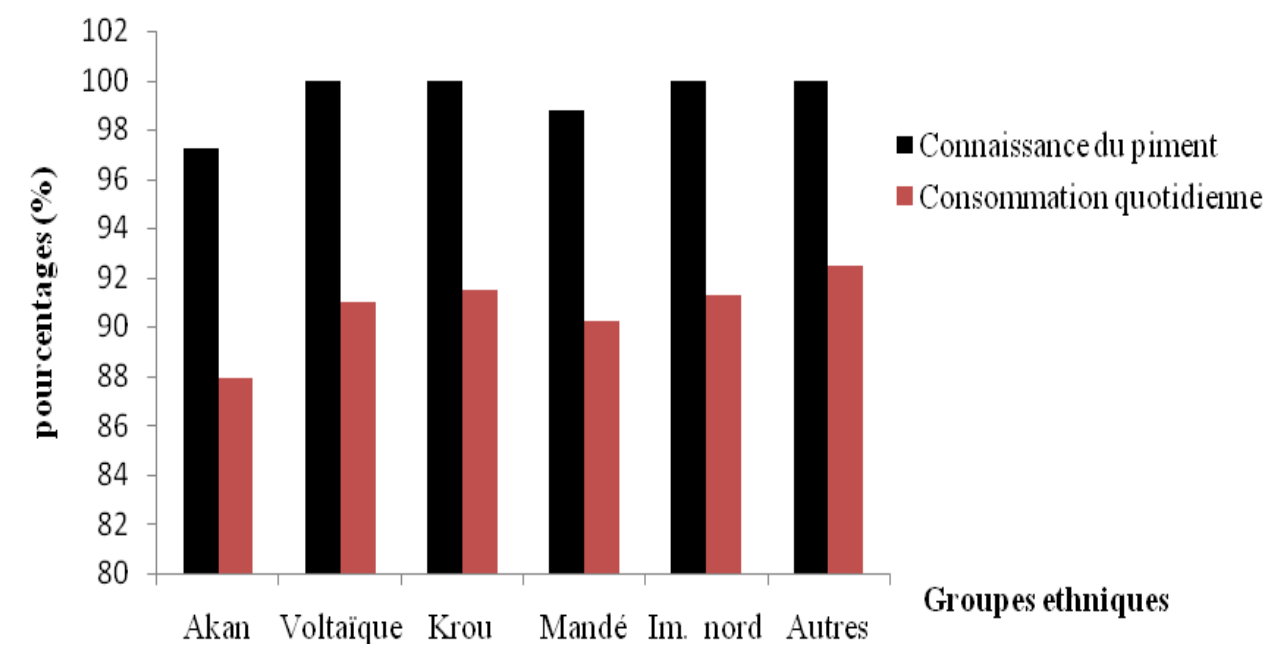

Figure 2 : Répartition de la connaissance et de la consommation du piment selon les groupes ethniques. 


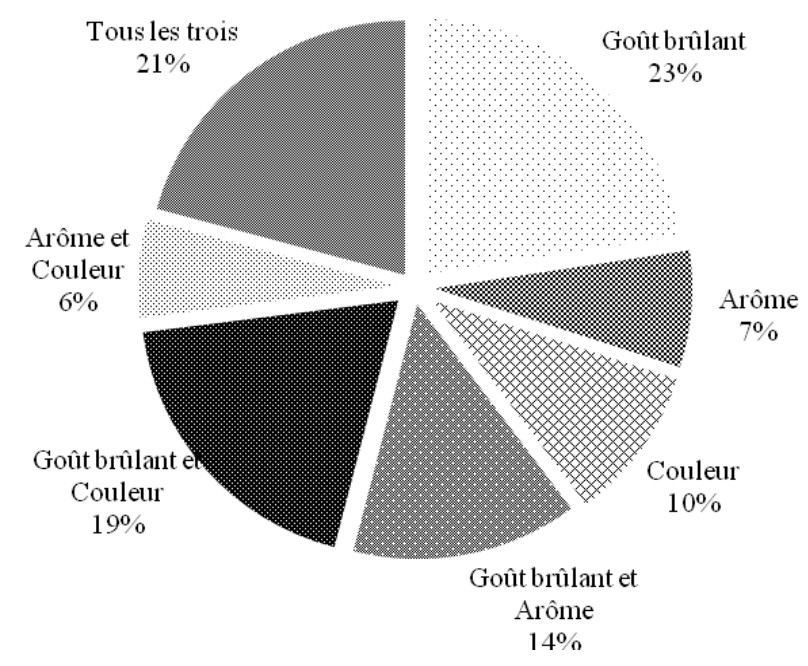

Figure 3 : Répartition de la consommation selon les propriétés sensorielles du piment.

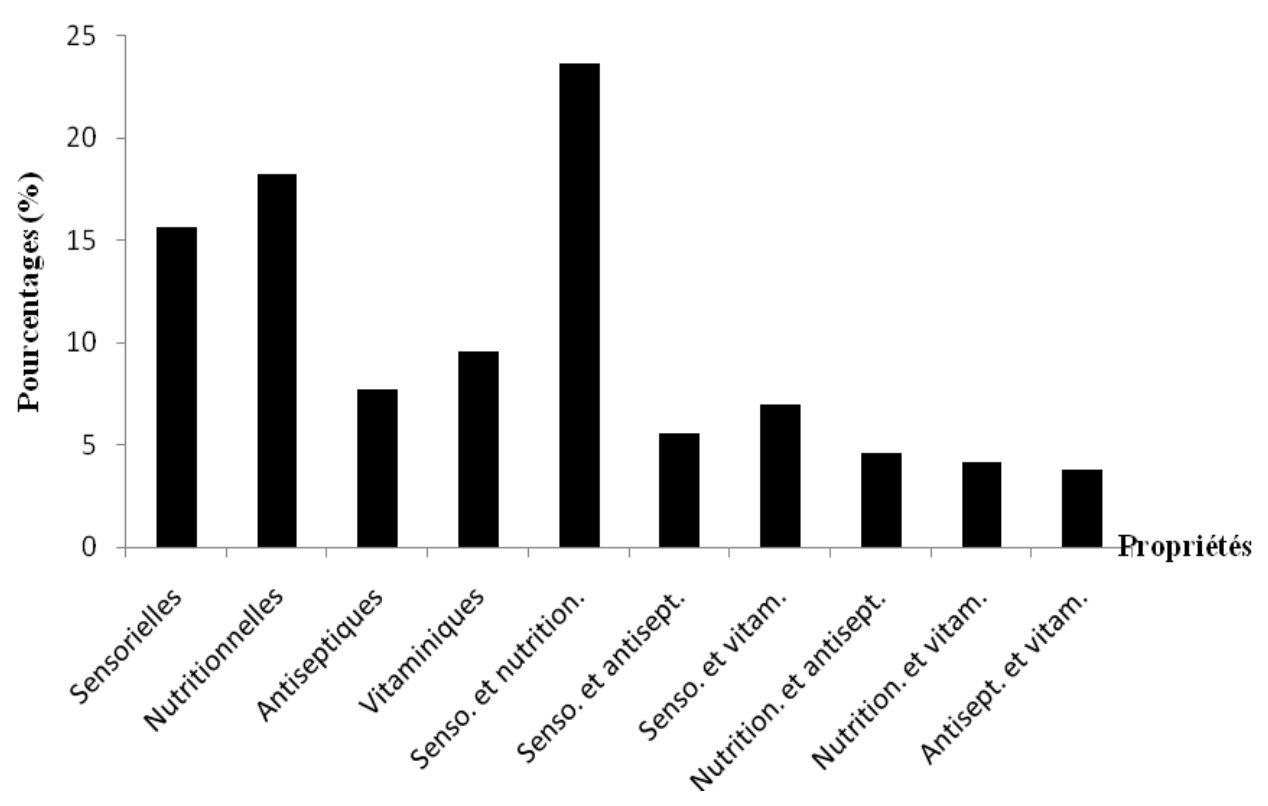

$($ Senso $=$ Sensorielles $;$ nutrition $=$ nutritionnelles $;$ vitam $=$ vitamines $;$ Antisept $=$ antiseptiques

Figure 4 : Répartition des propriétés soutenant la consommation du piment. 


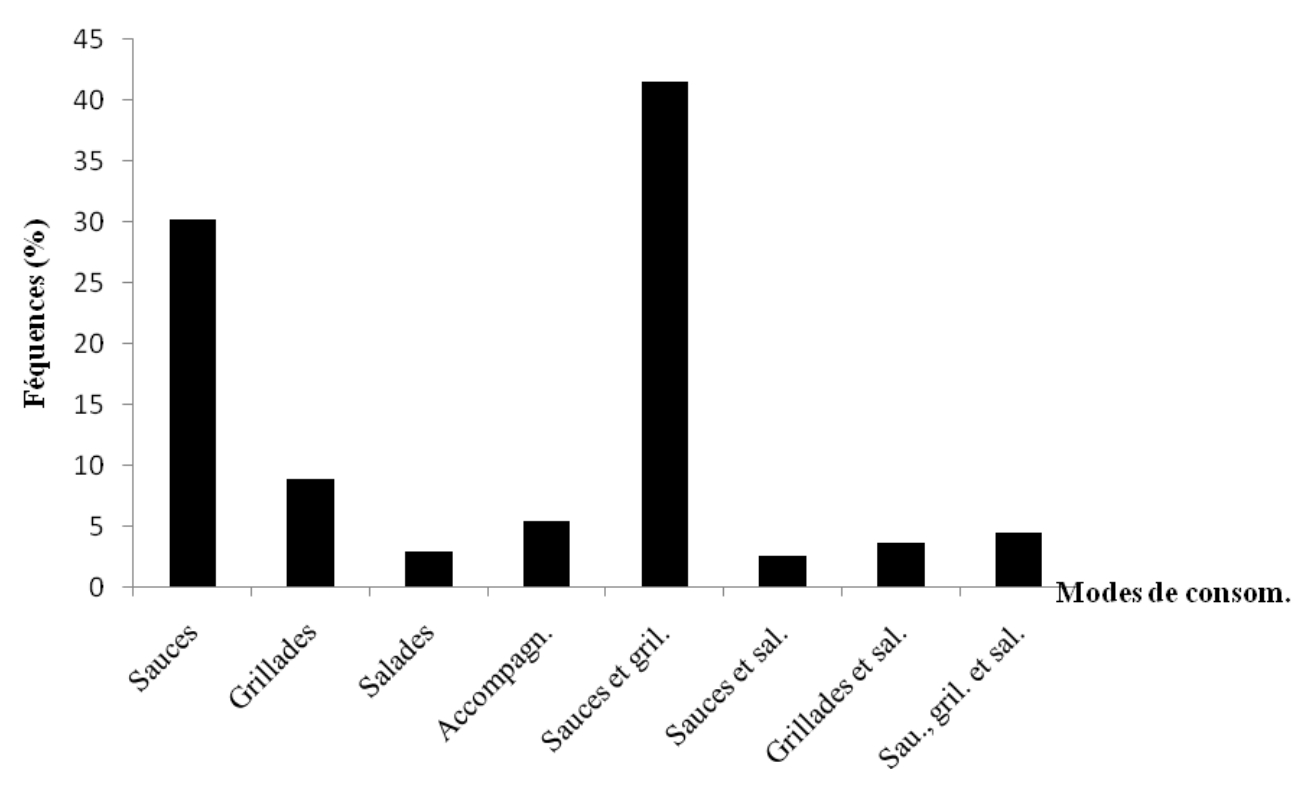

$($ Accompagn.$=$ Accompagnement $;$ gril.$=$ grillades $;$ sal. $=$ salades $;$ Sau.$=$ Sauce $)$

Figure 5 : Modes de consommation du piment.

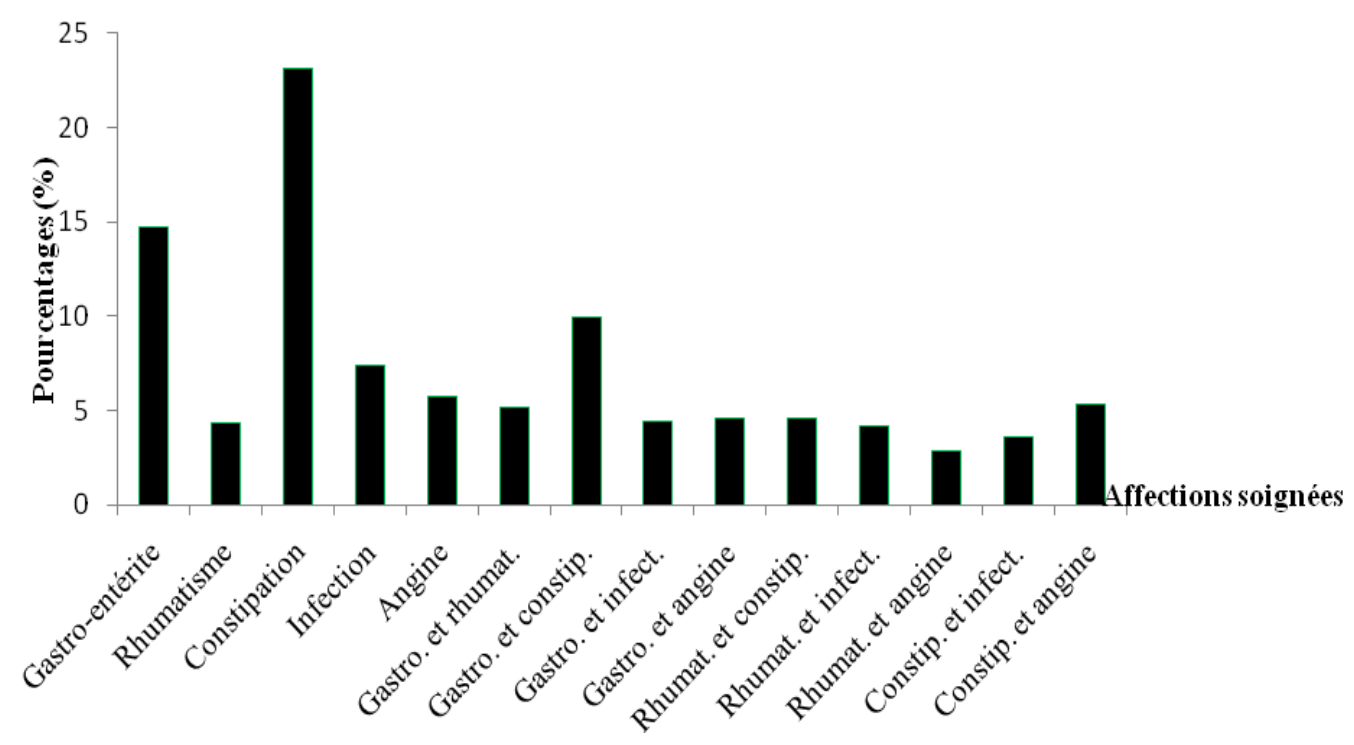

$($ Gastro $=$ Gastro-entérite $;$ rhumat $=$ rhumatisme $;$ constip $=$ constipation $;$ infect $=$ infection $)$

Figure 6 : Repartition des affections soignées par le piment. 


\section{DISCUSSION}

Le piment utilisé comme condiment ou épice est très bien connu en Côte d'Ivoire aussi bien par les femmes, les hommes que par les différents groupes ethniques. Cette observation confirme celle de Fett (2003) qui a indiqué que les piments sont les épices et les condiments les plus connus. Capsicum annuum et Capsicum frutescens font partis des espèces de piment généralement citées dans la littérature (Menichini et al. (2009). Ces résultats confirment ceux de HervertHernandez et al. (2010) qui stipulent que ces espèces de piment ont un large éventail de composés phytochimiques aux propriétés antioxydantes bien connues.

Le piment est plus consommé par les femmes $(97,16 \%)$ que par les hommes $(87,65 \%)$. Ces résultats confirment ceux de Amiot-Carlin et al. (2007) et Blanck et al. (2008) qui indiquent que les fruits et légumes sont plus consommés par les femmes. Les différents groupes ethniques consomment régulièrement le piment. Ce qui est en accord avec ceux de Amiot-Carlin et al. (2007) qui ont relevé l'importance de l'origine ethnique (hispanique, asiatique ou afro-américaine) dans la consommation des légumes à travers une étude américaine. Ces résultats confirment également ceux de RodriguezBurruezo et al. (2010) et aussi ceux de Jonhson (2007). Ces derniers ont évoqué la place du piment dans la cuisine indienne et son importance croissante dans les restaurants ethniques d'Europe. Le choix préférentiel des légumes dans l'alimentation a été signalé dans d'autres études (Zimmer et al., 2012).

Les principaux modes culinaires du piment demeurent les sauces et les grillades qui sont caractéristiques des peuples africains qui ont une préférence pour les plats chauds (cuits). L'une des principales raisons de la consommation du piment est le goût piquant et irritant qui donne une sensation de chaleur dans la bouche. L'effet de chaleur dans un repas serait un attribut de qualité de valeur importante qui se traduit par la salivation, la stimulation de l'appétit et les goûts qu'il relève. Il faut noter que la couleur d'un fruit à maturité est aussi captivante que sa saveur, et que dans l'art culinaire, la couleur a un grand pouvoir d'appétence sur les consommateurs (Bauer et al., 2010). Capsicum Antillais et Jaune sont de couleur verte et deviennent respectivement rouge et jaune à maturité avec un goût poivré ou piquant, alors que Capsicum frutescens Doux passe du blanc cassé au rouge lorsque mûri et ne possède pas de goût poivré. Les résultats de cette étude sur la forme et les propriétés sensorielles des différentes variétés de piment sont en accord avec ceux de Zimmer et al. (2012). En effet, ces derniers ont indiqué que la taille, la forme, la saveur et la chaleur sensorielle des fruits de Capsicum varient énormément.

La capsaïcine, alcaloïde lipophilique et principal composé responsable du goût poivré du piment ne serait pas liée à la couleur, et aurait un effet positif sur le mécanisme d'excrétion (Collera-Zuniga et al., 2004 ; Kollmannsberger et al., 2011. Les résultats des travaux de Pino et al. (2007) ont montré que la teneur en capsaïcine varie selon la composition en composés volatiles des différentes variétés. L'arôme, le parfum ou l'odeur d'un fruit stimule toujours sa consommation. Ces résultats soutiennent ceux de Avaloz De La Cruz (2007) qui, dans une analyse sensorielle a montré que la couleur, le goût piquant, et l'arôme des fruits de Capsicum frais ou cuits étaient des descripteurs qui avaient une influence positive sur l'acceptation générale d'un aliment 
(Johnson, 2007; Kollmannsberger et al., 2011).

La constipation, les gastro-entérites et les angines sont les affections les plus traitées par le piment. les populations continuent d'exploiter le pouvoir guérissant des plantes dont le piment pour traiter diverses maladies (Kappel et al., 2008 ; Meghvansi et al., 2010). Les formes d'emplois du piment dans la médecine traditionnelle sont en premier les lavements, suivis de l'ingestion de décoctions, les emplâtres et enfin les pansements (Kouassi et al., 2012). D'après les travaux de Jonhson (2007) et ceux de N'guessan et al. (2009), les ingrédients dérivés de Capsicum sont utilisés dans plusieurs produits cosmétiques à des concentrations aussi élevées que $5 \%$. Plusieurs travaux ont montré le pouvoir antimicrobien des extraits de piment sur certaines bactériens pathogènes telles $S$. aureus, E. coli, Bacillus, Vibrio, Pseudomonas et Clostridium (De Marino et al., 2008 ; Kappel et al., 2008 ; Meghvansi et al., 2010, Mueller et al., 2010).

Ce travail, premier du genre a permis de faire une analyse descriptive sur la connaissance, la consommation et les usages culinaires et médicinaux des variétés de piments cultivées et consommées en Côte d'Ivoire. Le piment est connu quel que soit le sexe et le groupe ethnique, comme exhausteur de goût des aliments. Il apporte une valeur ajoutée aux propriétés organoleptiques des mets tels les sauces et les grillades. Les informations recueillies permettront d'établir une base de données sur le piment et contribueront à faire de ce fruit-légume, une ressource agricole à prendre en compte dans tout programme de valorisation des richesses floristiques dont disposent la Côte d'Ivoire.

\section{REFERENCES}

Amiot-Carlin MJ, Caillavet F, Causse M, Combris P, Dallongeville J, Padilla M, Renard C, Soler LG. 2007. Les fruits et légumes dans l'alimentation. Enjeux et déterminants de la consommation. Expertise scientifique collective, synthèse du rapport, INRA (France). 80 p.

Avaloz De La Cruz DA. 2007. Faisabilité de la production au Mexique de fromages de chèvre additionnés de piment: aspects technologiques, sensoriels, sanitaire et économique. Thèse de Doctorat. Institut national polytechnique de Lorraine. Lorraine, France. 273p.

Blanck HM, Gillespie C, Kimmons JE, Seymour JD, Serdula MK. 2008. Trends in fruit and vegetable consumption among U.S. men and women, 1994-2005. Prev Chronic Dis., 5: A35.

Bauer W, Badou R, Loliger J, Etournaud A. 2010. Science et Technologie des Aliments: Principes de Chimie des Constituants et Technologie des Procédés. Ed. Presses Polytechniques et Universitaires Romandes ; 720p.

Collera-Zuniga O, Jimenez, FG, Gordollo RM. 2004. Comparative study of carotenoid composition in three Mexican varieties of Capsicum annuum L. Food Chemistry, 90: 109-114.

De Marino S, Iorizzi M, Zollo F. 2008. Antioxidant activity and biological properties of phytochemicals in vegetables and spices (Capsicum, Laurus, Foeniculum). Electronic Journal of Environmental, Agricultural and Food Chemistry, 7(10): 3174-3177.

FAO (Food and Agriculture Organization of the United Nations) 2006. Statistiques Alimentaire. Accessed www. FAO.org. Dernière mise à jour Mars 2006. 
Fett DD. 2003. Botanical briefs: Capsicum pepper. Cutis, 72(1): 21-3.

Hervert-Hernandez D, Sayago-Ayerdi SG, Goni I. 2010. Bioactive compounds of four hot pepper varieties (Capsicum annuum L.): antioxidant capacity, and intestinal bioaccessibility. Journal of Agricultural and Food Chemistry, 58: 3399-3406.

Johnson W Jr.2007. Final report on the safety assessment of Capsicum annuum extract, Capsicum annuum fruit extract, Capsicum annuum resin, Capsicum annuum fruit powder, Capsicum frutescens fruit, Capsicum frutescens fruit extract, Capsicum frutescens resin, and capsaicin. Int J Toxicol., 26(1): 3-106.

Jouzier E. 2005. Solanacées médicinales et philatélie. Bulletin de la Société de Pharmacie de Bordeaux, 144: 311-332.

Kappel VD, Costa GM, Scola G, Silva FA, Landell MF, Valente P, Souza DG, Vanz DC, Reginatto FH, Moreira JC. 2008. Phenolic content and antioxidant and antimicrobial properties of fruits of Capsicum baccatum L. var. pendulum at different maturity stages, Journal of Medicinal Food, 11: 267-274.

Kollmannsberger H, Rodríguez-Burruezo A, Nitz S, Nuez F. 2011. Volatile and capsaicinoid composition of ají (Capsicum baccatum) and rocoto (Capsicum pubescens), two Andean species of chile peppers. J. Sci. Food Agric., 91(9):1598-611.

Kouassi CK, Koffi-Nevry R, Nanga ZY, Teixeira Da Silva JA, Yao K, Lathro JS, Tano K, Loukou GY. 2010. Assessing the antibacterial activity and phytochemical screening of Capsicum varieties from Côte d'Ivoire. Food, 4(1): 27-32.
Kouassi C. 2012. Potentialités bioactives et activité antimicrobienne des variétés de piment (Capsicum) cultivées en Côte d'Ivoire. Thèse de Doctorat; Université d'Abobo-Adjamé, Abidjan, Côte d'Ivoire, $161 \mathrm{p}$.

Ludy MJ, Mattes RD 2011. The effects of hedonically acceptable red pepper doses on, thermogenesis and appetite. Physiol Behav., 102(3-4):251-258.

Meghvansi MK, Siddiqui S, Khan MH, Gupta VK, Vairale MG, Gogoi JK, Singh L. 2010. Naga chilli: a potential source of capsaicinoids with broad-spectrum ethnopharmacological applications. Journal of Ethnopharmacology, 132: 114.

Menichini F, Tundis R, Bonesi M, Loizzo MR, Conforti F, Statti G, De Cindio B, Houghton PJ, Menichini F 2009. The influence of fruit ripening on the phytochemical content and biological activity of Capsicum chinense Jacq. cv Habanero. Food Chemistry, 114: 553560.

Mueller M, Hobiger S, Jungbauer A. 2010. Anti-inflammatory activity of extracts from fruits, herbs and spices. Food Chemistry, 122: 987-996.

N'guessan K, Kadja B, Zirihi G, Traoré D, Aké-Assi L. 2009. Screening phytochimique de quelques plantes médicinales ivoiriennes utilisées en pays Krobou (Agboville, Côte-d'Ivoire) Sciences \& Nature, 1: 1 - 15.

Pino J, Gonzalez M, Ceballos L, CenturionYah AR, Trujillo-Aguirre J, LatournerieMoreno L, Sauri-Duch E. 2007. Characterization of total capsaicinoids, colour and volatile compounds of Habanero chilli pepper (Capsicum chinense Jack.) cultivars grown in 
Yucatan. Food Chemistry, 104: 16821686.

Rodríguez-Burruezo A, González-Mas Mdel C, Nuez F. 2010. Carotenoid composition and vitamin A value in ají (Capsicum baccatum $\mathrm{L}$.) and rocoto (C. pubescens $\mathrm{R}$. $\&$ P.), 2 pepper species from the Andean region. J. Food Sci., 75(8): S446-453.

Tano K, Koffi-Nevry R, Koussémon M, Oulé M K. 2008. The effects of different storage temperatures on the quality of fresh Bell pepper (Capsicum annum L.). Agricultural Journal, 3(2): 157-162.

Zimmer AR, Leonardi B, Miron D, Schapoval E, Oliveira JR, Gosmann G. 2012. Antioxidant and anti-inflammatory properties of Capsicum baccatum: From traditional use to scientific approach. $J$. Ethnopharmacol., 139 (1): 228-233. 